\title{
Is Virtual Reality Uniquely Effective in Eliciting Empathy?
}

\begin{abstract}
It has been argued that virtual reality is uniquely effective at eliciting empathy, because it is the best technology for putting an audience "into the shoes" of another person. Is there really a difference between a virtual reality experience and reading an article when it comes to inducing empathy? In this experimental study, we gave participants a pre-test and post-test survey regarding their empathy, attitudes and intentions toward immigrants, refugees and endangered animals. Between the surveys, randomly selected participants engaged with a virtual reality experience relating to the U.S.-Mexican border, and others read an article on the same topic. A virtual reality experience increased participants' empathy toward immigrants, led to more positive attitudes toward immigration and increased the participants' stated likelihood of taking political action in favor of immigrants. However, in almost all cases these increases were statistically indistinguishable from the increase due to reading a print article. No evidence was found for a strong, diffuse effect of virtual reality beyond the specific subject of the virtual reality experience itself.
\end{abstract}

Keywords: empathy, compassion, virtual reality, immigration, attitudes

Word count: 6267

Jason J. Jones *

Department of Sociology and Institute for Advanced Computational Science

Stony Brook University

Stony Brook, New York 11794-4356

Telephone: (631) 632-7700

Fax: (631) 632-8203

Jason.J.Jones@stonybrook.edu

ORCID\# 0000-0002-4140-0268

* corresponding author

Jamie M. Sommer

Department of Sociology

Stony Brook University

Stony Brook, New York 11794-4356

Telephone: (631) 632-7700

Jamie.Sommer@stonybrook.edu 


\section{Introduction}

After spending the past few decades as "vaporware" - an exciting new technology that has not yet materialized - virtual reality (VR) devices are now being mass-manufactured and delivered. As social scientists, we must address what impact this new medium will have on the social world, and its potential benefits for society at large. A frequently made claim is that VR will increase pro-social behavior because it is uniquely effective at eliciting empathy (Milk, 2015; Bailenson, in press).

Empathy involves the ability to understand others from their point of view, which includes thoughts, feelings, and behaviors that may be different from one's own (Baron-Cohen \& Wheelwright, 2004). Empathizing with others enables us to anticipate their feelings and behaviors, allowing us to respond appropriately and create relationships (Allison et al., 2011). Empathy includes both affective and cognitive responses (Batson et al., 1997). The former involves the ability to emotionally relate to others, while the latter involves the ability to understand how others are thinking and feeling (Batson, 2011). Both contribute to our ability to recognize how others are feeling and respond accordingly.

The relationships between empathy and social and political views have long been of interest to sociologists (Stephan and Finlay, 1999; Wagaman and Segal, 2014). For example, some studies find that those who have high empathy tend to feel responsible for the well-being of others, and as a result, work toward improving the lives of those in need (Morrell, 2010; Pinker 2011). People with higher levels of empathy are more prone to social activism, and call for government intervention to increase well-being (Hoffman, 2011; Wagaman and Segal, 2014). In particular, those who are highly empathetic are concerned with creating more equal societies and relationships (Chiao, 2010; Pratto et al., 1994), and tend to be more accepting and tolerant to 
people who are different from them (Baston et al., 1997; Dovidio et al., 2009). In short, increasing empathy may serve to heighten social consciousness, justice, and action (Keltner et al., 2010; Hoffman, 2011; Wagaman and Segal, 2014).

Based on this previous research, there is reason to believe that a VR intervention might be used to increase empathy toward a marginalized group and that changes in attitudes and intended actions would necessarily follow. Further, it would be useful to compare the effects of a VR intervention to an intervention in a more common medium - namely, print. Therefore, we conducted an experiment to test the potential impact of VR and print stimuli on empathy, attitudes and actions.

To do so, a sample of 50 participants were given a pretest about their views and feelings toward several current political issues, including Mexican immigration. The questionnaire consisted of items regarding empathy toward certain targets, attitudes about those targets and intended political actions. Participants were randomly assigned to one of 2 groups: one group read a New York Times article related to the Mexican-American border and the other was presented the Times' companion virtual reality experience. After treatment, participants completed an identical posttest.

Before moving on to our specific hypotheses, we review recent research on VR and empathy. Following this review, we explain in detail the research design.

\section{Prior Work}

VR experiences are thought to have unique potential to increase empathy because they can be used to replace the user's normal sensory environment with that from a new perspective (Milk, 2015; Bailenson, in press). It is argued VR thus has exceptional power in helping the user enter an unfamiliar world and exist in the context of another. 
For example, Formosa et al. (2017) found that exposure to VR simulations of schizophrenic symptoms increased empathy scores from pretest to posttest. The authors recommend that such simulations be used to teach tolerance and acceptance for those with psychiatric disorders.

Other studies have found initial support for psychological effects of a VR intervention. Working with children, Horace et al. (2016) tested six different VR experiences aimed at improving behaviors associated with Autism Spectrum Disorders (ASD). They found that VR was effective as a social training device, yielding improvements on various measures. Robitaille et al. (2016) investigated how participants with and without post-traumatic brain injuries responded to VR avatar simulations. The researchers used a sample of 12 people in the military, 6 with injury and 6 without in a controlled experiment. Based on their findings, they argue that VR simulated avatars can treat military personal with mild post-traumatic brain injury.

However, these studies focused on psychological health outcomes rather than the social implications of VR use. Studies that do focus on social outcomes are either hypothetical, or measure small-scale social "helping behavior," such as picking up a researcher's dropped pens. For example, Keating (2017) proposes a study where video material is collected to capture the journey from Syria to outside of the country, and then transformed into a VR experience. Such material would ideally capture the experiences of a Syrian refugee. The researcher hopes that once this VR experience is created that it will help improve empathy for Syrian refugees among its viewers.

Rosenberg et al. (2013) used a controlled experiment with 60 student participants (30 male and 30 female). The participants were broken into 4 groups, and each of the groups experienced one of these scenarios: their avatar flying around a city in a helicopter to help a 
child, their avatar flying around a city in a helicopter just for a tour, their avatar flying around a city like a superhero to help a child, or their avatar flying around the city like a superhero just for a tour. After the VR experience, participants responded to questionnaire items including motion sickness questions, how real the experience felt, the Prosocial Orientation Questionnaire, and what they thought the purpose of the experiment was. Respondents were also exposed to a researcher dropping pens to see if those who experienced different conditions would be more likely to pick up the pens. It was observed that those who experienced flying like a superhero were more likely to pick up the researcher's pens than those who were in the helicopter regardless of the task. The authors argue that emulating a superhero flight increased empathetic behavior.

In sum, there seems to be growing support for and interest in the idea that VR can elicit empathy in its users. Outside of academia, organizations are not waiting on scientific evidence; they are acting on beliefs that VR has unique benefits. For example, in Australia, an unnamed company sponsored a virtual reality homelessness experience for CEOs in conjunction with a charity fundraising event (CEO Sleepout, 2017). The production company Within (formerly VRSE) collaborated to produce a VR experience with the non-profit Charity: Water, and the organizations claim the experience influenced over $\$ 2.4$ million in donations (Swant, 2016).

Research and applied use of VR is in its infancy - just as the widespread availability of the technology is itself. In this work, we set out to test the idea that VR is uniquely effective at eliciting empathy, and if so, how that translates to attitude change and intentions to act.

\section{Hypotheses}

\section{$\underline{V R}$ and Empathy}


Studying virtual reality in a controlled experiment presents a unique opportunity to observe and direct empathy and test hypotheses regarding how it is best elicited. The first hypothesis we aim to test is that of a direct effect of VR on empathy:

$\mathrm{H1}$ : A virtual reality experience that is focused on the topic of immigration will increase participant empathy toward immigrants.

Any discussion of VR and empathy is premature without first testing for the existence of a basic relationship between the stimulus and the anticipated response. There is broad belief that media consumption affects emotion and cognition (Scheufele \& Tewksbury, 2007). As another medium, we would expect VR to do so as well.

Our second hypothesis is that the effect of VR on empathy is not only positive, but uniquely so, because of the intensity of the sensory experience:

$\mathrm{H} 2$ : A virtual reality experience that is focused on the topic of immigration will increase participant empathy toward immigrants more than reading an article on the same topic.

The argument from intuition goes as such: Imagine you read about the plight of a Mexican immigrant. Now contrast that to virtually experiencing the immigrant's hardships for yourself. Clearly in the second case you would gain a deeper appreciation and understanding, in the same way that attending a funeral would provide more detail and context than reading about it in a newspaper obituary. In short, a virtual experience is full of detail and subtlety that must be elided in a written account.

Another possibility is that virtual reality is an experience beyond that provided by any previous medium. Philippe Bertrand, an artist who has used virtual reality has said, "It's disorienting - an experience you have never had in your life" (quoted in Alsever, 2015). Jeremy 
Bailenson, director of Stanford University's Virtual Human Interaction Lab, has echoed those remarks: "We are entering an era that is unprecedented in human history, where you can transform the self and experience anything" (ibid.) If VR is a medium of a new kind, then perhaps it may transform the sense of self entirely. Rather than a focused, specific effect (e.g. a virtual reality experience of homelessness increases one's empathy towards the homeless) what

if the experience of having one's perception so altered as to be virtually placed in a new reality causes a diffuse, general effect? Our third hypothesis addresses this potential general effect: H3: A virtual reality experience will increase empathy generally among participants.

\section{$\underline{V R}$ and Attitudes}

We also seek evidence that a VR experience can do more than engender personal empathy. In particular, we aim to test the proposition that attitudes toward the subject of a VR experience shall change as well.

H4: A virtual reality experience that is focused on the topic of immigration will increase the positivity of participant attitudes toward immigrants.

The above hypothesized effect would be especially interesting if VR experiences were distinct from other media in their consequence. Imagining another's perspective based on a written document is an active process requiring cognitive effort. It is easy to believe some consumers would not bother or have the resources to do so. A VR experience, in contrast, can be consumed passively with less effort. Indeed, it intrudes into one's environment by way of a headset enveloping the eyes and headphones covering the ears. We aim to test the idea that the greater immersion of the VR experience will lead to a larger effect. 
H5: A virtual reality experience that is focused on the topic of immigration will increase the positivity of participant attitudes toward immigrants more than reading an article on the same topic.

There is also the possibility that a VR experience is so immersive that the effect may generalize and change a respondent's attitudes toward other groups. Peck, Seinfeld, Aglioti, and Slater (2014) found that among a group of 60 female participants, those who received darkskinned avatars compared to light-skinned or other-skinned avatars had more improved Implicit Association Test (IAT) scores from pretest to posttest. The researchers suggested that similar VR experiences would be helpful in reducing bias in general. This idea is captured in the hypothesis of a general effect of VR interventions:

H6: A virtual reality experience will increase the positivity of attitudes generally among participants, even for targets not mentioned in the experience.

\section{$\underline{V R}$ and Actions}

Several recent studies indicate VR has the potential to alter behaviors (Formosa et al.

2017; Horace et al. 2016; Marco, Perpina, \& Botella, 2013). In this study, we expect that:

H7: A virtual reality experience that is focused on the topic of immigration will increase participants'stated likelihood of taking actions meant to benefit immigrants.

Again, there is reason to expect that reading an article has a different effect on measurable actions than having a VR experience. For instance, Ahn, Bailenson, and Park (2014) using an immersive virtual environment found that those who "cut" a tree down consumed $20 \%$ less paper than participants who read about "tree cutting." The authors conclude that such virtual 
treatments may help encourage conservation behaviors. Similarly, we shall test the following hypothesis:

H8: A virtual reality experience that is focused on the topic of immigration will increase participants' stated likelihood of taking actions meant to benefit immigrants more than reading an article on the same topic.

For the sake of symmetry with our previous sets of hypotheses, we shall also test for a general effect of the VR experience on actions regarding other issues. It would certainly be worth noting if a VR experience regarding one specific topic produced a greater desire to take political actions generally.

H9: A virtual reality experience will increase participants'stated likelihood of taking political actions in general.

As a subset of intended actions, we also measured hypothetical intentions to share resources with target groups and organizations. Participants were asked to distribute $\$ 100$ (in hypothetical money) among money they wished to keep and money they would donate to Mexican immigrants, Syrian refugees or the protection of whales.

\section{Method}

\section{$\underline{\text { Participants }}$}

50 undergraduates attending Stony Brook University participated in the study.

\section{$\underline{\text { Design }}$}

The design was 2 (Test Timing) x 2 (Media Modality) mixed design. Test Timing refers to a pretest which preceded the manipulation and a posttest which followed. All subjects completed both the pretest and the posttest. Media Modality refers to the stimulus provided to 
the participant. Half of the subjects were randomly assigned to read an article and the other half to engage with a VR experience.

\section{$\underline{\text { Stimuli }}$}

We sought a VR experience that had a substantially similar written complement. We found both by making use of existing material. The New York Times recently began producing virtual reality experiences to supplement their reporting, and we evaluated several before choosing an article and experience relating to the U.S.-Mexican border.

Specifically, the media stimuli for this experiment were a VR experience and article both entitled "10 Shots Across the Border." The article can be found at the URL: https://www.nytimes.com/2016/03/06/magazine/10-shots-across-the-border.html. The virtual reality experience can be found at the URL: http://www.nytimes.com/marketing/nytvr/. To engage with the experience in the same way the participants did, one should download the "NYT VR" app on an iPhone or Android smartphone.

\section{Procedure}

Participants were run through the protocol one at a time in a room with only the experiment materials and the experimenter. The experimenter greeted the participant and directed them to a swivel chair facing a tablet computer resting on a table. The participant read a consent form on the computer and indicated consent.

Next, participants gave their responses to the pretest. Most items required a response indicating the participant's level of agreement with a statement. All of the pretest items were repeated on the posttest, and the posttest is included in Appendix A in this manuscript. For each of three target subjects, the participants responded to items regarding empathy, attitudes, intended actions and resource sharing. The three subjects each had a general and a specific 
instantiation. Namely, the specific category of Mexican immigrants corresponded to the general category of immigrants; Syrian refugees to refugees and whales as the specific category for the environment.

Between the pretest and posttest, the experimental manipulation was applied. Half of the participants engaged with the virtual reality experience, and the other half read the article. The VR experience has a runtime of about eight minutes. Participants used an iPhone 6, headphones and a Google Cardboard viewer. Participants were seated in a swivel chair and were free to move their heads and bodies as they liked. The application tracked movements and changed perspective in accordance. For example, in the VR experience, the participant was encouraged to look down while "flying" in a helicopter. At another point, the participant was prompted to look behind themselves at a border wall.

Participants in the Article condition were given 10 minutes to read a printed version (free of advertisements) of the VR experience companion article. Finally, the participants responded to the posttest. An additional set of demographic and interest items followed the items on the posttest that were repeated from the pretest.

\section{Results}

In this manuscript, we discuss statistical tests referring specifically to the hypotheses introduced above. We conduct analyses pre-registered before viewing the data. For the analysis plan and analysis making use of additional variables, see the online material at https://osf.io/jdma8/.

\section{Empathy}

All responses to empathy items referring to immigrants were scored $0,1,2,3$, and 4 on a Likert scale. To illustrate, if a subject chose "Strongly Disagree" as a response to the statement 
"I relate to Mexican immigrants" that would be scored 0. If they responded "Strongly Agree" this was scored 4 . The responses to empathy items regarding immigrants were summed to form a composite Immigrant Empathy scale. This process remains the same for responses for attitudes and actions, and the process was repeated separately for refugee and environment items. Figure 1 summarizes the results for Immigrant Empathy.

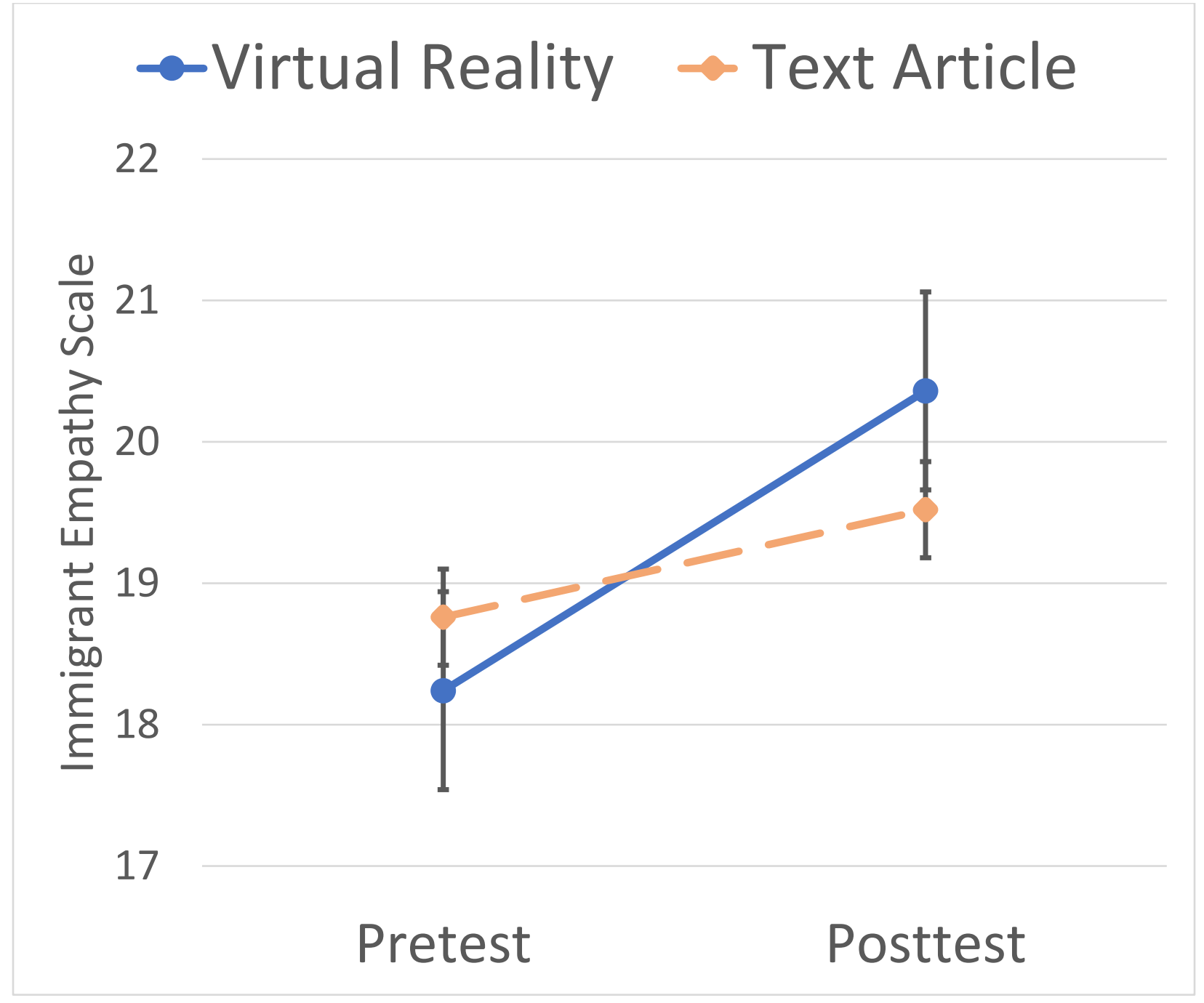

Figure 1. Immigrant Empathy scale total at pretest and posttest by Media Modality. Media Modality varied between participants. Pretest and posttest consist of the same items and scores are compared within participant. Error bars represent the within-subject standard error. 
To test H1, we employ a one-tailed, paired t-test contrasting VR participants' pretest responses to the immigrant empathy items to the same participants' posttest responses. This test supports a positive effect of VR on empathy $\underline{\mathrm{t}}(24)=2.13, \underline{\mathrm{p}}<0.05$.

To test H2, we employ a one-tailed, independent samples t-test contrasting the difference scores (D-scores) of the VR subject group and the Article subject group. This test indicates marginal at best support for the proposition that the increase in Immigrant Empathy is larger for VR participants as opposed to Article subjects $\underline{\mathrm{t}}(48)=1.23, \underline{\mathrm{p}}=0.11$.

An ANOVA conducted on the entire design grid confirms the previous results. The main effect of Test Timing on Immigrant Empathy is significant $\underline{F}(1,48)=6.77, \underline{p}<0.05$. No main effect of Media Modality was observed. An interaction in the predicted direction of $\mathrm{H} 2$ did not reach significance $\underline{\mathrm{F}}(1,48)=1.51, \underline{\mathrm{p}}=0.23$.

To test H3, we employ two one-tailed, paired t-tests contrasting VR participants' pretest responses to the refugee and environment empathy items to the same participants' posttest responses. These tests provide ambiguous evidence. On the one hand, empathy toward environmental targets appears to increase $\underline{\mathrm{t}}(24)=2.98, \underline{\mathrm{p}}<0.05$. On the other, empathy toward refugees does not increase $\underline{\mathrm{t}}(24)=-1.99, \underline{\mathrm{p}}=0.97$.

\section{$\underline{\text { Attitudes }}$}

Figure 2 illustrates the results for Immigrant Attitudes. To test H4, we employ a onetailed, paired t-test contrasting VR participants' pretest responses to the immigrant attitude items to the same participants' posttest responses. This test supports a positive effect of the VR intervention on attitudes toward immigrants $\underline{\mathrm{t}}(24)=1.95, \underline{\mathrm{p}}<0.05$.

To test H5, we employ a one-tailed, independent samples t-test contrasting the difference scores (D-scores) of the VR subject group and the Article subject group. This test supports the 
hypothesis that the positive effect of VR was greater than the effect of the article $t(48)=2.04, \mathrm{p}$ $<0.05$.

An ANOVA produces results consistent with those above. Neither main effect is significant, however the interaction indicates the positive effect of the VR intervention differs from the effect of the article $\underline{F}(1,48)=4.14, \underline{p}<0.05$.

To test H6, we employ two one-tailed, paired t-tests contrasting VR participants' pretest responses to the refugee and environment attitude items to the same participants' posttest responses. Neither test produced a significant difference.

\section{$\rightarrow$-Virtual Reality $\rightarrow$ Text Article}

40
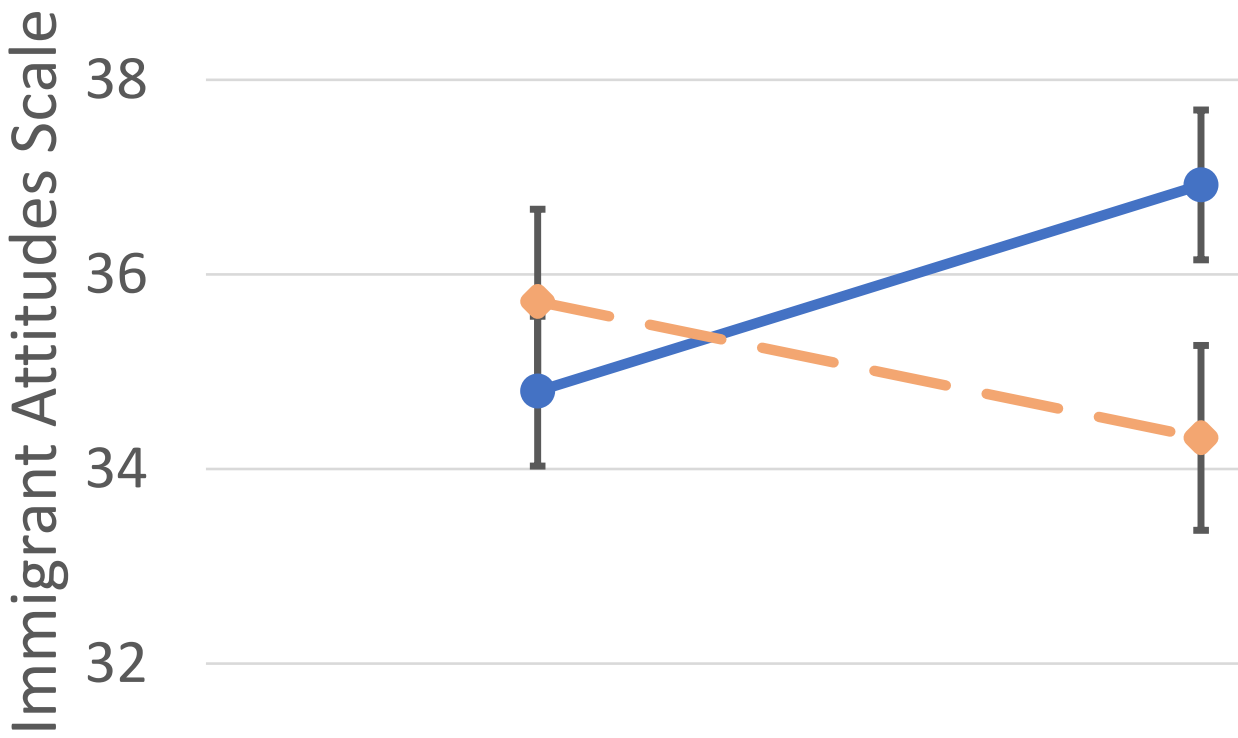

30 
Figure 2. Attitude scale total at pretest and posttest by Media Modality. Media Modality varied between participants. Pretest and posttest consist of the same items and scores are compared within participant. Error bars represent the within-subject standard error.

Actions

Figure 3 illustrates the results for the immigrant intended actions scale. To test H7, we employ a one-tailed, paired t-test contrasting VR participants' pretest responses to the immigration intended action items to the same participants' posttest responses. This test supports a positive effect of VR on Action $\underline{\mathrm{t}}(24)=2.16, \underline{\mathrm{p}}<0.05$.

To test H8, we employ a one-tailed, independent samples t-test contrasting the difference scores (D-scores) of the VR subject group and the Article subject group. This test did not indicate a significant difference.

To test H9, we employ two one-tailed, paired t-tests contrasting VR participants' pretest responses to the refugee and environment action items to the same participants' posttest responses. Neither test produced a significant difference. 


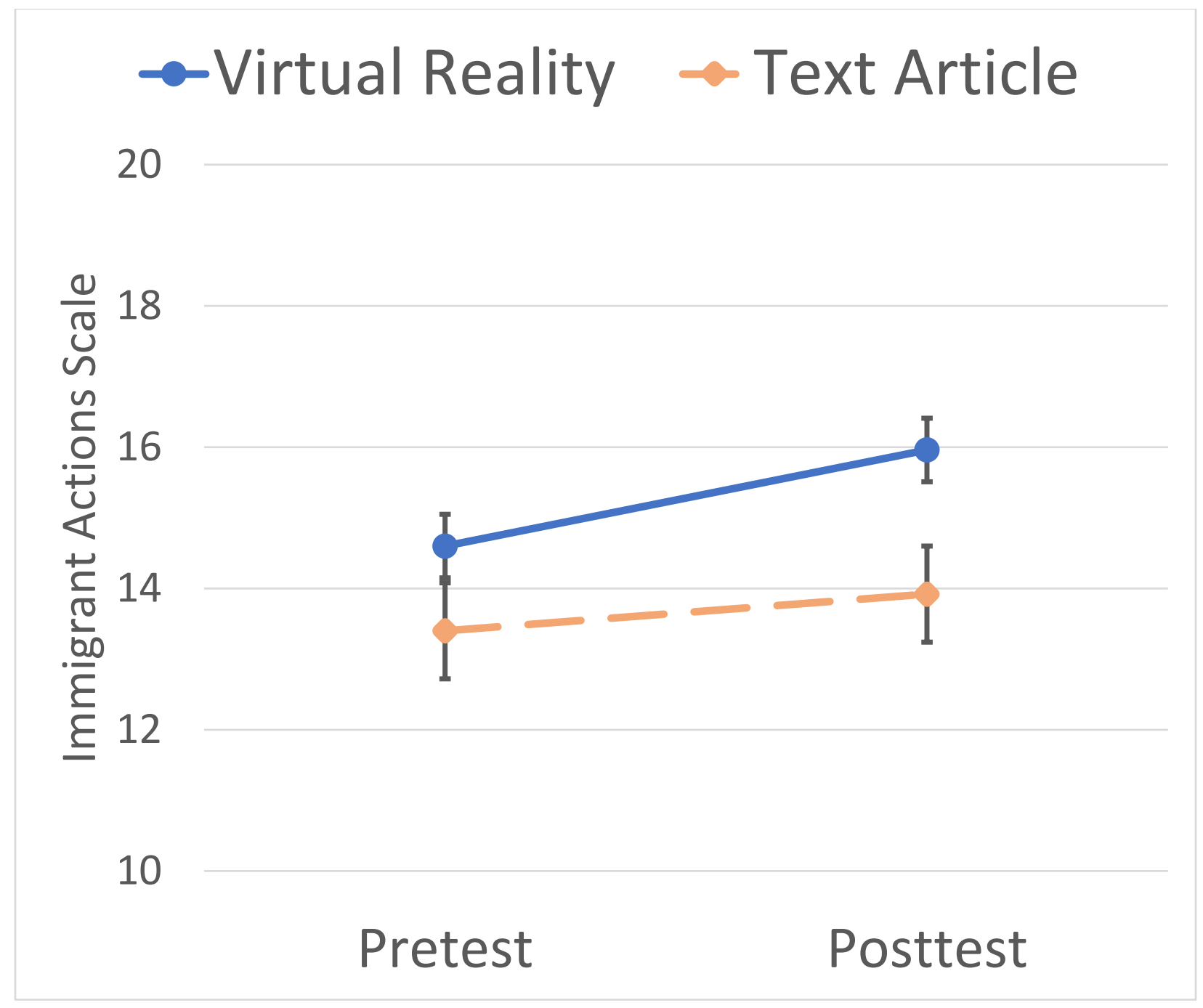

Figure 3. Action scale total at pretest and posttest by Media Modality. Media Modality varied between participants. Pretest and posttest consist of the same items and scores are compared within participant. Error bars represent the within-subject standard error.

Figure 4 displays results for a second Action measure: hypothetical resource sharing. The response plotted here is the mean dollar amount directed to Mexican immigrants as a function of experimental condition. 


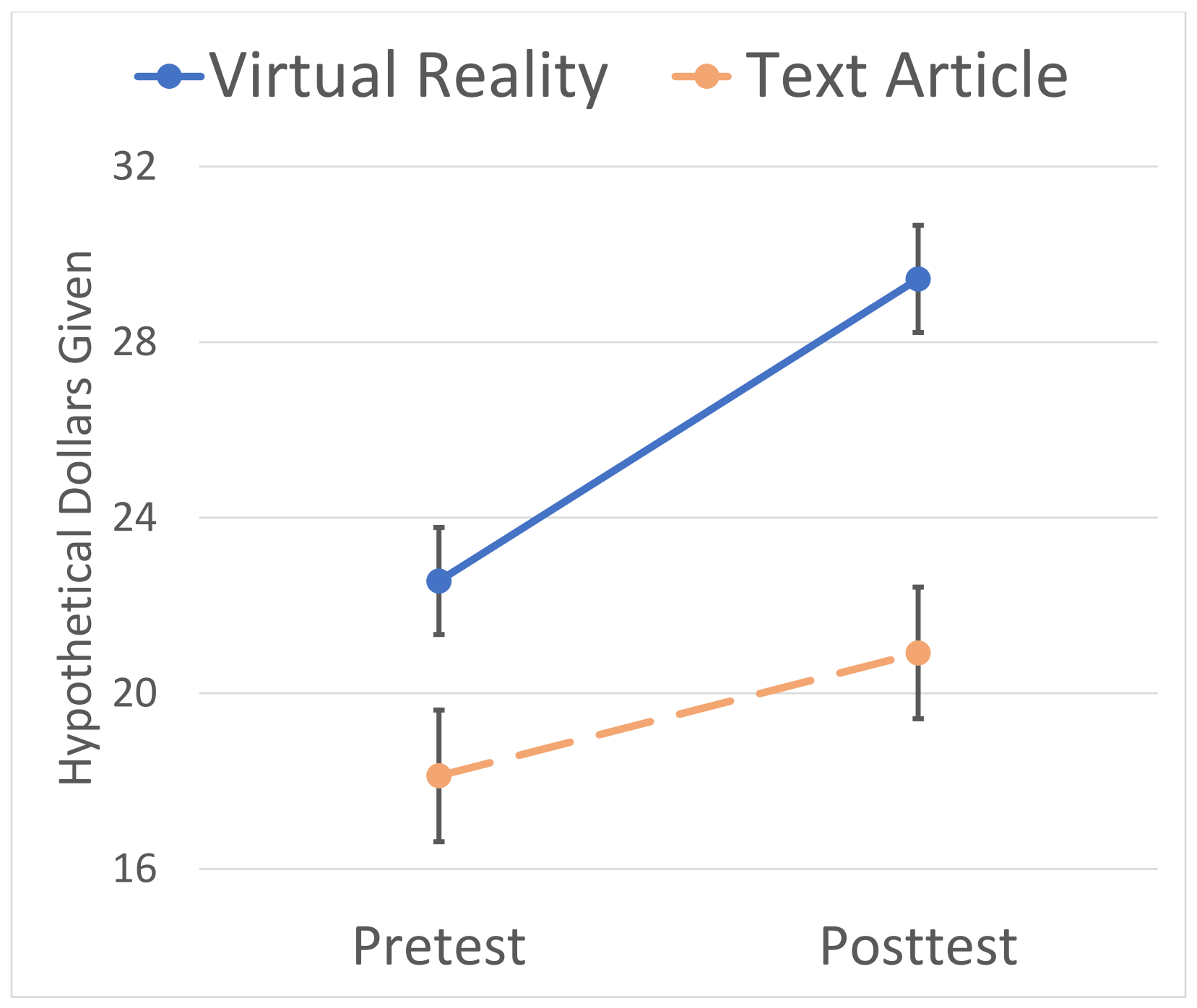

Figure 4. Dollars directed toward Mexican immigrants at pretest and posttest by Media Modality. Error bars represent the within-subject standard error.

An ANOVA indicates results similar to those for the Immigrant Empathy scale. The main effect of Test Timing is significant $\underline{\mathrm{F}}(1,48)=4.59, \underline{\mathrm{p}}<0.05$. No main effect of Media Modality was observed. An interaction in the predicted direction of $\mathrm{H} 8$ did not reach significance $\underline{F}(1,48)$ $=1.56, \underline{p}=0.22$.

\section{Discussion and Conclusion}


A VR experience regarding the U.S.-Mexican border increased empathy with immigrants. However, the evidence is inconclusive as to whether this effect is larger for a VR experience as opposed to reading an article. The evidence for a general effect of VR on empathy beyond the specific subject of the experience is also equivocal.

A valid interpretation of these results would be that spending a mere eight minutes in a VR experience increased empathy by 12 percent (i.e. the mean score on the immigrant empathy scale moved from 18.2 to 20.4). This may explain the enthusiasm proponents of the new medium feel. Seeing a new perspective truly does change one's feeling of connection with another.

But it remains entirely plausible that a text-only article printed on paper had the same effect. A great book (or article) engages the imagination and "takes us there" in a virtual manner that may be as good or better for the purpose of empathizing as the virtual reality experience enabled by marvels of digital technology.

Proponents of the medium certainly act as if use of VR promotes empathy. The results presented here demonstrate they are not misguided. The brief VR intervention we employed not only increased empathy felt toward immigrants, but also led to participants favoring less restrictive immigration policies and indicating they would be more likely to take political actions to the benefit of immigrants. The same caveat regarding media modality applies, however. With one exception, the effects of a VR experience and a written article were statistically indistinguishable.

The possibility hinted at by the diverging slopes of the lines in Figures 1 through 4 is too important to dismiss with a shrug. A differential effect may be real, but not dramatic enough to be revealed with the limited statistical power of a 50-participant experiment. If VR is the 
"ultimate empathy machine" some claim, news media, entertainment and societies should prepare for disruption. Who will wield the power to choose the targets of your empathy and to which ends?

One possibility we can rule out is that of a strong, diffuse effect of VR pertaining to all subjects and not just the content of the VR experience itself. The VR intervention's effects were mostly constrained to the topic of the experience - immigration - and did not spill over into general attitude change or intended political activity.

As authors, we have deliberately chosen to phrase the title of this manuscript as a question rather than a statement. Clearly there is value in using virtual reality experiences to increase human compassion. Whether it is a radically new and efficient way to do so remains to be seen. 


\section{References}

Ahn, S. J. G., Bailenson, J. N., \& Park, D. (2014). Short-and long-term effects of embodied experiences in immersive virtual environments on environmental locus of control and behavior. Computers in Human Behavior, 39, 235-245.

Allison, C., Baron-Cohen, S., Wheelwright, S. J., Stone, M. H., \& Muncer, S. J. (2011). Psychometric analysis of the Empathy Quotient (EQ). Personality and Individual Differences, 51(7), 829-835.

Alsever, J. (2015). Is Virtual Reality the Ultimate Empathy Machine? Retrieved August 7, 2017, from https://www.wired.com/brandlab/2015/11/is-virtual-reality-the-ultimate-empathymachine/

Bailenson, J. (in press). Experience on Demand: What Virtual Reality Is, How It Works, and What It Can Do (1 edition). New York, NY: W. W. Norton \& Company.

Baron-Cohen, S., \& Wheelwright, S. (2004). The empathy quotient: an investigation of adults with Asperger syndrome or high functioning autism, and normal sex differences. Journal of autism and developmental disorders, 34(2), 163-175.

Batson, C. D., Polycarpou, M. P., Harmon-Jones, E., Imhoff, H. J., Mitchener, E. C., Bednar, L. I., Klein, T. R., \& Highberger, L. (1997). Empathy and attitudes: Can feeling for a member of a stigmatized group improve feelings toward the group? Journal of Personality and Social Psychology, 72(1), 105-118.

Batson, C.D. (2011). These things called empathy: Eight related but distinct phenomena. In J. Decety \& W. Ickes (Eds.), The social neuroscience of empathy (pp. 3-15). Cambridge, MA: MIT Press. 
CEO Sleepout. (2017). New technology to educate. Retrieved August 7, 2017, from https://www.ceosleepout.org.au/newtechtoeducate/

Chiao, J.Y. (2010). Neural basis of social status hierarchy across species. Current Opinion in Neurobiology, 20, 803-809.

Dovidio, J. F., Gaertner, S. L., \& Saguy, T. (2009). Commonality and the complexity of "we": Social attitudes and social change. Personality and Social Psychology Review, 13, 3-20.

Formosa, N. J., Morrison, B. W., Hill, G., \& Stone, D. (2017). Testing the efficacy of a virtual reality-based simulation in enhancing users' knowledge, attitudes, and empathy relating to psychosis. Australian Journal of Psychology.

Hoffman, M. L. (2011). Is empathy necessary for morality? In A. Coplan \& P. Goldie (Eds), Empathy: Philosophical and psychological perspectives (pp. 231-254). New York: Oxford University Press.

Horace, H. S. IP., Wong, S. W., Chan, D. F., Byrne, J., Li, C., Yuan, V. S., \& Wong, J. Y. (2016). Virtual reality enabled training for social adaptation in inclusive education settings for school-aged children with autism spectrum disorder (ASD). In International Conference on Blending Learning (pp. 94-102). Springer International Publishing.

Keating, B. (2017). Changing Hearts and Minds, using Virtual Reality to Improve Empathy towards Refugees. Prehospital and Disaster Medicine, 32(S1), S86-S86.

Keltner, D., Marsh, J., \& Smith, J. A. (2010). The compassionate instinct. New York: WW Norton \& Company.

Marco, J. H., Perpina, C., \& Botella, C. (2013). Effectiveness of cognitive behavioral therapy supported by virtual reality in the treatment of body image in eating disorders: one year follow-up. Psychiatry Research, 209(3), 619-625. 
Morrell, M. E. (2010). Empathy and democracy: Feeling, thinking, and deliberation. University Park, PA: Pennsylvania State University

Milk, C. (2015). How virtual reality can create the ultimate empathy machine. Retrieved from https://www.ted.com/talks/chris_milk_how_virtual_reality_can_create_the_ultimate_emp athy_machine.

Peck, T. C., Seinfeld, S., Aglioti, S. M., \& Slater, M. (2013). Putting yourself in the skin of a black avatar reduces implicit racial bias. Consciousness and cognition, 22(3), 779-787.

Pinker, S. (2011). The better angels of our nature: Why violence has declined. New York: Viking.

Pratto, F., Sidanius, J., Stallworth, L. M., \& Malle, B. F. (1994). Social dominance orientation: A personality variable predicting social and political attitudes. Journal of Personality and Social Psychology 67(4), 741-763.

Robitaille, N., Jackson, P. L., Hébert, L. J., Mercier, C., Bouyer, L. J., Fecteau, S., \& McFadyen, B. J. (2016). A Virtual Reality avatar interaction (VRai) platform to assess residual executive dysfunction in active military personnel with previous mild traumatic brain injury: proof of concept. Disability and Rehabilitation: Assistive Technology, 1-7.

Rosenberg, Robin S., Shawnee L. Baughman, and Jeremy N. Bailenson. (2013). Virtual superheroes: Using superpowers in virtual reality to encourage prosocial behavior. PloS one 8(1), e55003.

Scheufele, D. A., \& Tewksbury, D. (2007). Framing, Agenda Setting, and Priming: The Evolution of Three Media Effects Models. Journal of Communication, 57(1), 9-20. https://doi.org/10.1111/j.0021-9916.2007.00326.x 
Stephan, W. G., \& Finlay, K. (1999). The role of empathy in improving intergroup relations. Journal of Social Issues, 55, 729-743.

Swant, M. (2016). How Virtual Reality Is Inspiring Donors to Dig Deep for Charitable Causes. Retrieved August 7, 2017, from http://www.adweek.com/digital/how-virtual-realityinspiring-donors-dig-deep-charitable-causes-171641/

Wagaman, M. A., \& Segal, E. A. (2014). The relationship between empathy and attitudes toward government intervention. J. Soc. \& Soc. Welfare, 41, 91. 


\section{Appendix A}

VR Post-Test

Please indicate your level of agreement with the below statements (strongly agree, agree, neither agree nor disagree, disagree, or strongly disagree).

I relate to Syrian refugees

I have a lot in common with Syrian refugees

I identify with Syrian refugees

I feel connected to Syrian refugees

I wish to feel more connected with Syrian refugees

I wish to better understand Syrian refugees

I wish to better understand what Syrian refugees experience

I feel sorry for Syrian refugees

I wish you could do something to help Syrian refugees

Please indicate your level of agreement with the below statements (strongly agree, agree, neither agree nor disagree, disagree, or strongly disagree).

I relate to Mexican immigrants

I have a lot in common with Mexican immigrants

I identify with Mexican immigrants 
I feel connected to Mexican immigrants

I wish to feel more connected with Mexican immigrants

I wish to better understand Mexican immigrants

I wish to better understand what Mexican immigrants experience

I feel sorry for Mexican immigrants

I wish I could do something to help Mexican immigrants

Please indicate your level of agreement with the below statements (strongly agree, agree, neither agree nor disagree, disagree, or strongly disagree).

I relate to whales

I have a lot in common with whales

I identify with whales

I feel connected to whales

I wish to feel more connected with whales

I wish to better understand whales

I wish to better understand what whales experience

I feel sorry for whales

I wish I could do something to help whales 
Please indicate your level of agreement with the below statements (strongly agree, agree, neither agree nor disagree, disagree, or strongly disagree).

I think immigration restrictions in the United States should be relaxed

I think immigration restrictions in the world should be relaxed

I think there should be fewer restrictions on migrants coming to the United States

I think there should be fewer restrictions on migrants in general

I think Mexican immigration restrictions in the United States should be relaxed

I think Mexican immigration restrictions in the world should be relaxed

I think there should be fewer restrictions on Mexican migrants coming to the United States

I think there should be fewer restrictions on Mexican migrants in general

I think we should welcome more immigrants to the United States

I think the world should welcome more immigrants into their borders

I think we should welcome more Mexican immigrants to the United States

I think the world should welcome more Mexican immigrants into their borders

I think the United States should have open borders

I think all nations should have open borders

I think the United States should have open borders to Mexicans

I think all nations should have open borders for Mexicans

I think the United States should continue to build the U.S./Mexican border wall 
Q7 Please indicate your level of agreement with the below statements (strongly agree, agree, neither agree nor disagree, disagree, or strongly disagree).

I think refugee restrictions in the United States should be relaxed

I think refugee restrictions in the world should be relaxed

I think there should be fewer restrictions on refugees coming to the United States

I think there should be fewer restrictions on refugees in general

I think we should welcome more refugees to the United States

I think the world should welcome more refugees into their borders

I think the United States should have open borders to refugees

I think all nations should have open borders to refugees

Q8 Please indicate your level of agreement with the below statements (strongly agree, agree, neither agree nor disagree, disagree, or strongly disagree).

I you think the United States needs to do more to protect whales

I think the United States needs to do more to protect the natural environment

I think the United States needs to do more to protect marine life

I think the United States could do more to protect whales

I think the United States could do more to protect the natural environment 
I think the United States could do more to protect marine life

I think the world needs to do more to protect whales

I think the world needs to do more to protect the natural environment

I think the world needs to do more to protect marine life

I think the world could do more to protect whales

I think the world could do more to protect the natural environment

I think the world could do more to protect marine life

Please respond how likely you are to do the indicated action (strongly agree, agree, neither agree nor disagree, disagree, or strongly disagree).

I will contact my US representative about Syrian refugees

I will contact my US representative about refugees

I will donate to Syrian refugee rights

I will donate to refugee rights

I will sign a petition about Syrian refugee rights

I will sign a petition about refugee rights

I will share information on social media about Syrian refugee rights (21)

I will share information on social media about refugee rights (22) 
Please respond how likely you are to do the indicated action (strongly agree, agree, neither agree nor disagree, disagree, or strongly disagree).

I will donate to environmental concerns

I will donate to habitat concerns of whales

I will contact my US representative about environmental concerns

I will contact my US representative about the habitat of whales

I will share information on social media about environmental concerns

I will sign a petition about environmental concerns

I will sign a petition about habitat concerns of whales

I will share information on social media about habitat concerns of whales

Please respond how likely you are to do the indicated action (strongly agree, agree, neither agree nor disagree, disagree, or strongly disagree).

I will sign a petition about Mexican immigrant rights

I will contact my US representative about Mexican immigrants

I will contact my US representative about immigrants

I will donate to Mexican immigrant rights

I will donate to immigration rights

I will sign a petition about immigration rights 
I will share information on social media about Mexican immigrant rights

I will share information on social media about immigration rights

Hypothetically, let's say we gave you $\$ 100$ to keep and/or give away. How would you allocate each dollar?

Whales

Syrian Refugees

Mexican Immigrants

Me (Yourself)

Hypothetically, let's say we gave you $\$ 100$ to keep and/or give away. How would you allocate each dollar to Charities and yourself?

Immigration Advocates: Non-Profit Resource Center

HIAN: Welcome the Stranger. Protect the Refugee

GreenPeace for the Natural Environment

Me (Yourself) 
Hypothetically, let's say we gave you $\$ 100$ to keep and/or give away. How would you allocate each dollar to Charities and yourself?

Southern Border Communities Coalition for Mexican Immigrants

Sunrise USA: The Leading Provider of Humanitarian Aid to Syrians in the US and

abroad

Whale and Dolphin Conservation Society

Me (Yourself)

What are your thoughts on what you learned and experienced today?

How frequently do you use social media sites like Facebook, Twitter, Instagram, etc.?

O Never

O Sometimes

O Daily 
How much do you care about politics?

None or very little

O A little

O Some

O A lot

O A whole lot

Do you think of yourself as more politically liberal, conservative, or neither?

L Liberal

O Conservative

O Neither

What gender do you identify with?

O Woman

O Man

O Genderqueer

Transgender

O Other 
What is your political affiliation?

Democrat

Republican

Independent

Other

What is your sex?

O Male

O Female

Intersex

Other

What is your level of education?

High School

Some college

2-year degree

4-year degree

Some graduate school

Graduate school

Other 
What is your age?

What is your parents' level of income?

Less than 19,999

20,000-49,999

50,000-79,999

O 80,000 or more

What is your race? For purposes of this question, persons of Spanish/Hispanic/Latino origin may be of any race.

Black

White

Asian / Pacific Islander

American Indian and Alaska Native

Other

Are you of Hispanic, Latino, or Spanish origin?

Y Yes

No 
Were you born in the United States of America?

O Yes

No

What country were you born in?

What is your ethnicity?

Please provide your email address so we can contact you in the future about this experiment.

If you used the virtual reality device, was this your first time using virtual reality?

O Yes

O No

Does not apply

Please indicate your level of agreement below (strongly agree, agree, neither agree nor disagree, disagree, or strongly disagree).

I am interested in using virtual reality

I am interested in using virtual reality in the future

I will use virtual reality in the future 